\section{Left ventricular pseudoaneurysm following acute myocardial infarction}

A 78-year-old man presented with severe chest distress and dyspnea for two days. He had been treated at a local hospital for ST-segment elevation myocardial infarction with acute anterior wall injury 20 days previously. Cardiac catheterization showed mild stenosis of the mid-left anterior descending branch of the coronary artery, and consequently a stent was not fitted. Transthoracic echocardiography findings revealed that the motion of the anterior apical wall of the left ventricle (LV) was reduced. He received thrombolytic and lipid-lowering therapy. After 1 week, the patient was discharged with diminished symptoms. His medical history included hypertension and emphysema. In the emergency room at our hospital, cardiovascular examination revealed a systolic-diastolic murmur in the apex. Echocardiography findings revealed a large apical pseudoaneurysm, which measured $76 \times 52 \mathrm{~mm}$ and was connected to the LV cavity via an apical wall defect (Fig. 1a, 1b; Videos 1, 2). Computed tomography (CT) with intravenous contrast confirmed the presence of a large apical pseudoaneurysm associated with the LV apex (Fig. 2). Arterial blood gas analysis indicated severe respiratory failure (type II). After discussing the patient's poor prognosis with his family, no further interventions were implemented. The patient died 2 weeks following the onset of symptoms.

A left ventricular pseudoaneurysm (LVP) is a cardiac rupture sealed by the pericardium and is a rare complication following transmural acute myocardial infarction. LVP has a high mortality rate. Echocardiography is crucial for prompt LVP diagnosis. A CT scan can delineate the extent of the LVP and the involvement of adjacent structures.

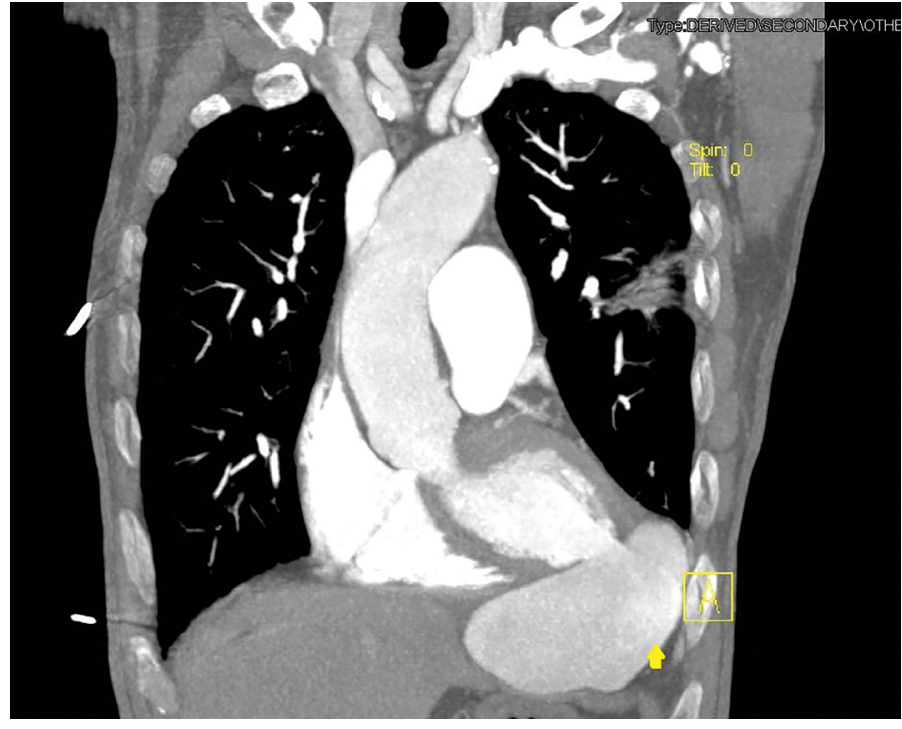

Figure 2. Computed tomography image of the heart showing a large left ventricular pseudoaneurysm (arrow)

Acknowledgements: The present study was supported by a grant from Chinese Medical Doctor Association. The funders played no role in study design, data collection and analysis, decision to publish, or preparation of the manuscript.

\section{(D) Wenjuan Bai, (D) Hong Tang \\ Department of Cardiology, West China Hospital of Sichuan University; Sichuan-China}

Video 1. Echocardiography in the apical four-chamber view showing a large left ventricular apical pseudoaneurysm measuring $76 \times 52 \mathrm{~mm}$ with a $15 \mathrm{~mm}$ neck connected with the left ventricle

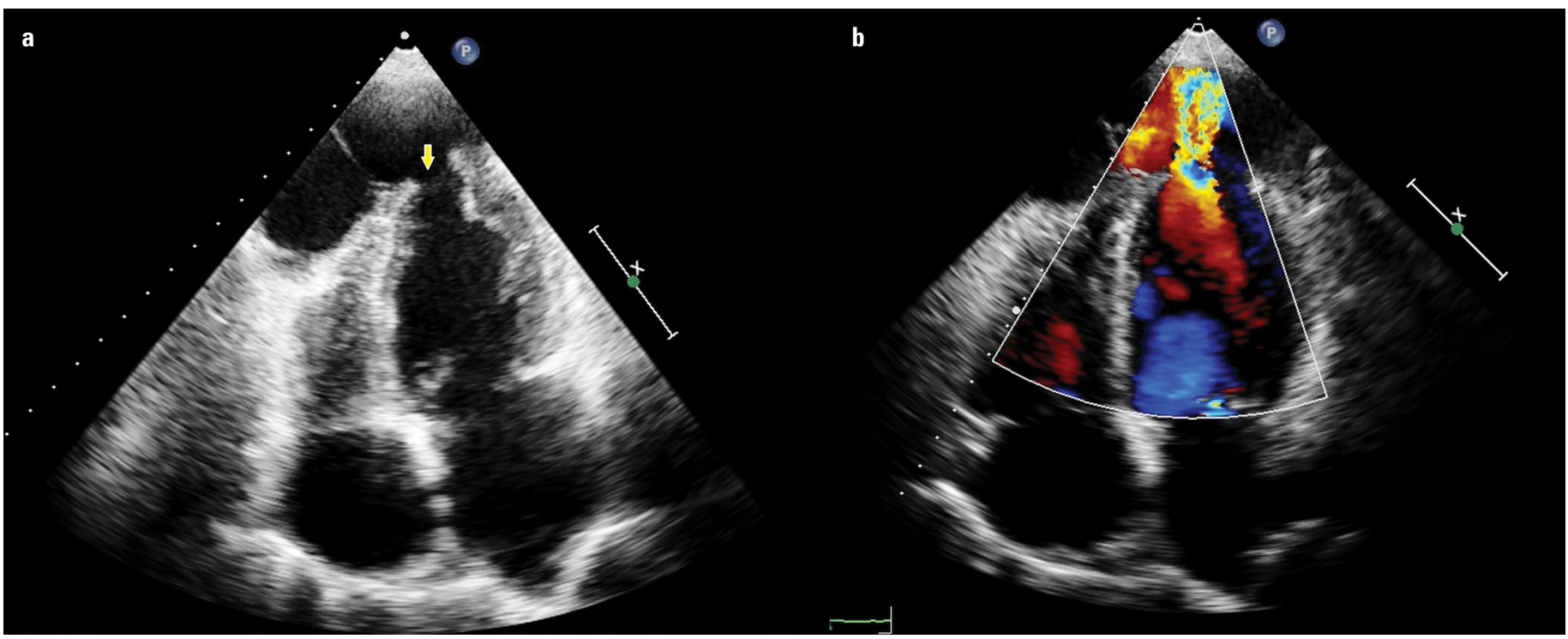

Figure 1. (a) Echocardiography in the apical four-chamber view showing a large left ventricular apical pseudoaneurysm measuring $76 \times 52 \mathrm{~mm}$ with a $15 \mathrm{~mm}$ defect (arrow) connected with the left ventricle. (b) Color-Doppler echocardiography confirming the association of the pseudoaneurysm and left ventricle 
Video 2. Color-Doppler echocardiography confirming the association of the pseudoaneurysm and left ventricle

Address for Correspondence: Wenjuan Bai, MD,

Department of Cardiology, West China Hospital of Sichuan University, 37 Guo Xue Xiang, Chengdu, 610041, Sichuan-China

Phone: 008618980860406

E-mail: littlewhitehx@126.com

(C) Copyright 2018 by Turkish Society of Cardiology - Available online

at www. anatolicardiol.com

DOI:10.14744/AnatolJCardiol.2018.39001 